Supporting Information

\title{
Live Cell MicroRNA Imaging Using Exonuclease III-Aided Recycling Amplification Based on Aggregation-Induced Emission Luminogens
}

Xuehong Min ${ }^{\ddagger}$, Mengshi Zhang ${ }^{\ddagger}$, Fujian Huang, Xiaoding Lou* and Fan Xia

Hubei Key Laboratory of Bioinorganic Chemistry and Materia Medica, School of Chemistry and Chemical Engineering, Huazhong University of Science and Technology, Wuhan, Hubei 430074, China

$\$$ X. M. \& M. Z. contribute equally. * Correspondence and requests for materials should be addressed to X. Lou. (Email: louxiaoding @ hust.edu.cn). 


\section{Experimental section}

\subsection{Materials}

Copper (I) bromide, sodium ascorbate and acetonitrile were purchased from Aladdin and used as received without further purification. Other chemicals were purchased from Sigma Aldrich and used as received without further purification. FAM-LDNA and Cy3-MBDNA were obtained from the DNA synthesis company, Takara in China. All of the cancer patient samples were donated by the First Affiliated Hospital of Nanchang University. The water used in the work is purified by a Millipore filtration system.

\subsection{Synthesis of TPEPy-DNA probe via the click reaction.}

We synthesized the TPEPy according to the reported reference. ${ }^{1}$ All the DNA sequences were obtained from the company, Takara in China. TPEPy-DNA was synthesized according to the process that used in our previous work. ${ }^{2}$ Scheme S1 showed the synthesis process for TPEPy-DNA.

\subsection{Fluorescence measurement}

The fluorescence detection was carried out on a Cary Eclipse Fluorescence Spectrophotometer (Agilent Technologies).

\subsection{Amplified detection of MicroRNAs}

We choose 1X Exonuclease III buffer as the reaction buffer. The Exonuclease III amplification was performed in $50 \mu \mathrm{L}$ of $1 \mathrm{X}$ Exonuclease III buffer. It contains 10 $\mu \mathrm{M}$ TPEPy-LDNA probe, $50 \mathrm{U}$ of Exonuclease III and corresponding concentrations of MicroRNAs. 


\subsection{MicroRNAs extraction from urine samples}

MicroRNAs were obtained from urine samples by using Norgen's Urine microRNA Purification Kit according to our previous work. ${ }^{2}$

\subsection{Cell culture and confocal microscopy imaging}

MCF-7 cells, and HeLa cells were obtained from Xiangya Central Experiment Laboratory (Hunan Province, China). Human lung fibroblast (HLF) cells were obtained from China Center (Hubei Province, China) for Type Culture Collection. HeLa and MCF-7 cells were maintained under the standard culture conditions (atmosphere of $5 \% \mathrm{CO}_{2}$ and $95 \%$ air at $37^{\circ} \mathrm{C}$ ) in RPMI 1640 medium, supplemented with $10 \%$ FBS (fetal calf serum) and $100 \mathrm{IU} / \mathrm{mL}$ penicillin-streptomycin. HLF cells were cultured in Minimum Essential Medium, MEM, plus 10\% FBS and $100 \mathrm{IU} / \mathrm{mL}$ penicillin-streptomycin in $5 \% \mathrm{CO}_{2}$ and $95 \%$ air at $37{ }^{\circ} \mathrm{C}$. HeLa, MCF-7 and HLF cells in the exponential phase were plated on $35 \mathrm{~mm}$ glass-bottom culture dishes $(\Phi$ $20 \mathrm{~mm}$ ) for 2 days to reach around $80 \%$ confluency respectively.

Cells were starved with $1 \mathrm{~mL}$ of serum-free medium Opti-MEM for $2 \mathrm{~h}$ before they were transfected with the detection probe (TPEPy-LDNA, FAM-LDNA, and Cy3-MBDNA) and Exonuclease III by liposome transfection. The transfection liquid solution was a mixture of two different solutions. The first one contained $10 \mu \mathrm{L}$ Lipofectamine 2000 Reagent and $140 \mu \mathrm{L}$ Opti-MEM, standing $5 \mathrm{~min}$ after fully mixing. The second one was added with the appropriate concentrations of enzyme and detection probes to make the total volume of $150 \mu \mathrm{L}$ Opti-MEM. The transfection liquid solution (300 $\mu \mathrm{L}$ total volume) was finally formed when the two solutions were kept in static for 15 min after fully mixing. Then the cells were incubated with the prepared transfection liquid solution which contained TPEPy-LDNA $(6.6 \mu \mathrm{M})$ and Exonuclease III $(1.33 \mathrm{U} / \mu \mathrm{L})$ in an atmosphere of $5 \% \mathrm{CO}_{2}$ and $95 \%$ air for $60 \mathrm{~min}$ at $37{ }^{\circ} \mathrm{C}$. The other two transfection liquid solution contained Cy3-MBDNA (500 nM) and Exonuclease III (1.33 U/ $\mu \mathrm{L})$ or FAM-LDNA (500 nM) and Exonuclease III (1.33 $\mathrm{U} / \mu \mathrm{L})$, respectively. And the transfection process was the same to that of 
TPEPy-LDNA. Cells were washed three times with $1 \mathrm{~mL}$ of PBS at room temperature and then they were added to $1 \mathrm{~mL}$ of PBS culture medium and observed under confocal microscopy (Olympus FV1200) with a 60xoil-immersion objective. To monitor the dynamic progress of the intracellular miR-21 in the corresponding cells, scanning was stopped after the completion of the first scanning picture. The light irradiation was kept for $10 \mathrm{~min}$ in situ before the following scanning. The scanning time interval was $10 \mathrm{~min}$ and the total light irradiation time was $60 \mathrm{~min}$. For Cy3-MBDNA, excitation was at $543 \mathrm{~nm}$ and emission collected was at 560-620 nm. For FAM-LDNA, excitation was at $488 \mathrm{~nm}$ and emission collected was at 510-540 nm. For TPEPy-LDNA, excitation was at 405nm and emission collected was at 560-620 nm. 


\section{Figures \& Tables}

\subsection{Organic synthesis of TPEPy-LDNA}
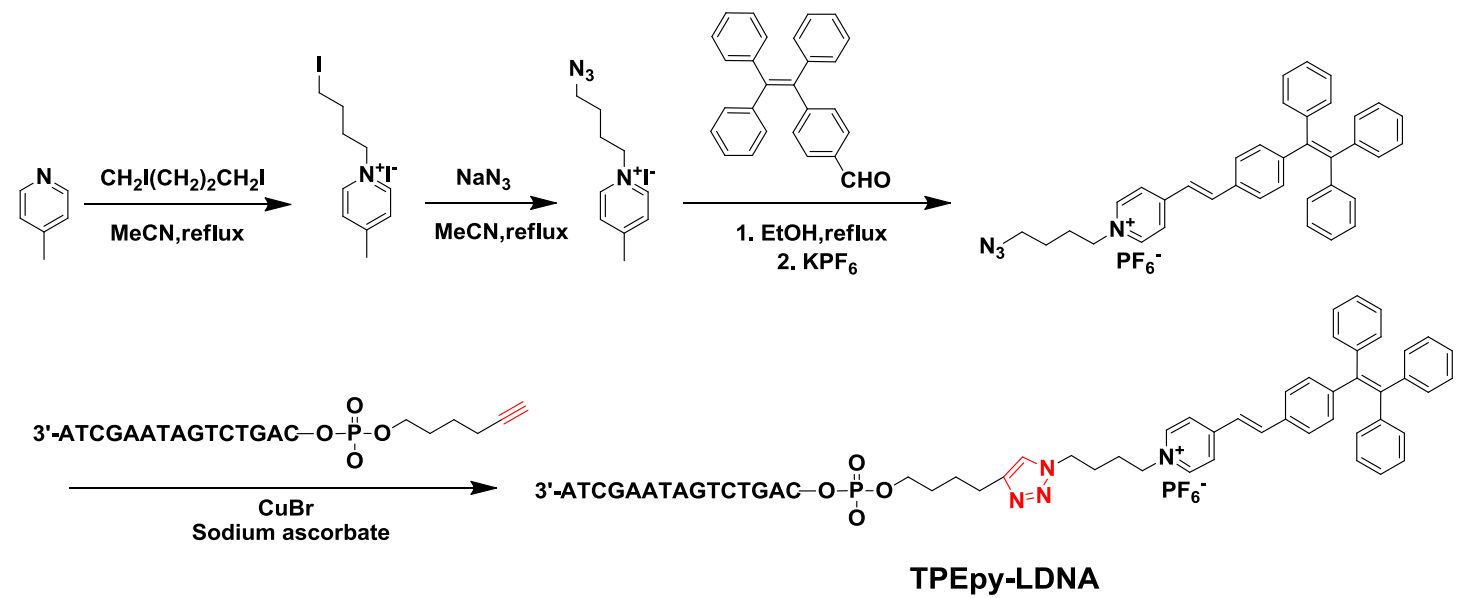

Figure S1. The organic synthesis of TPEPy-LDNA. 
2.2 Mass spectrum of TPEPy- $\mathrm{N}_{3}$

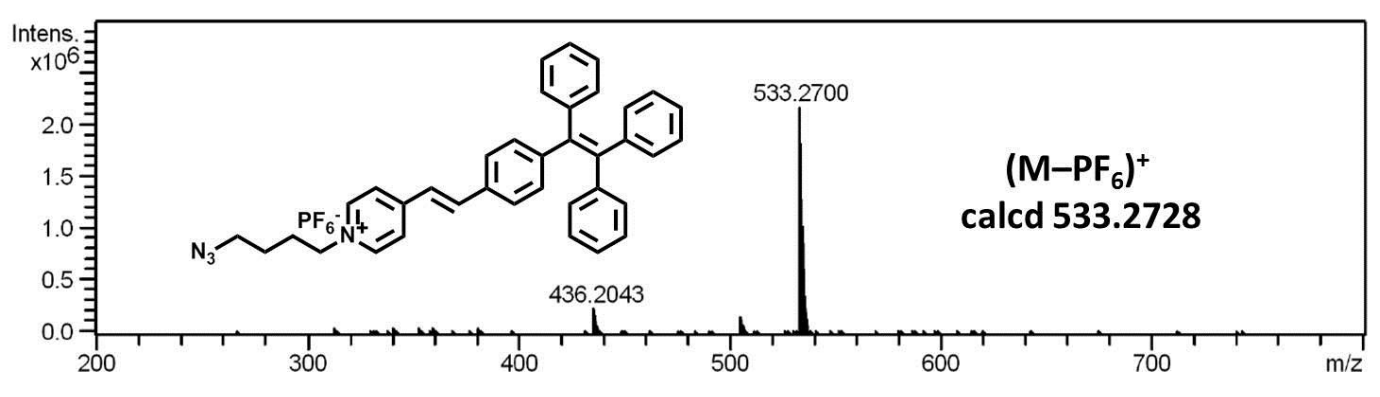

Figure S2. The Mass spectrum of TPEPy-N $\mathrm{N}_{3}$. It reveals molecular weight 533.27. 
2.3 Chemical structure and mass spectrum of TPEPy-LDNA

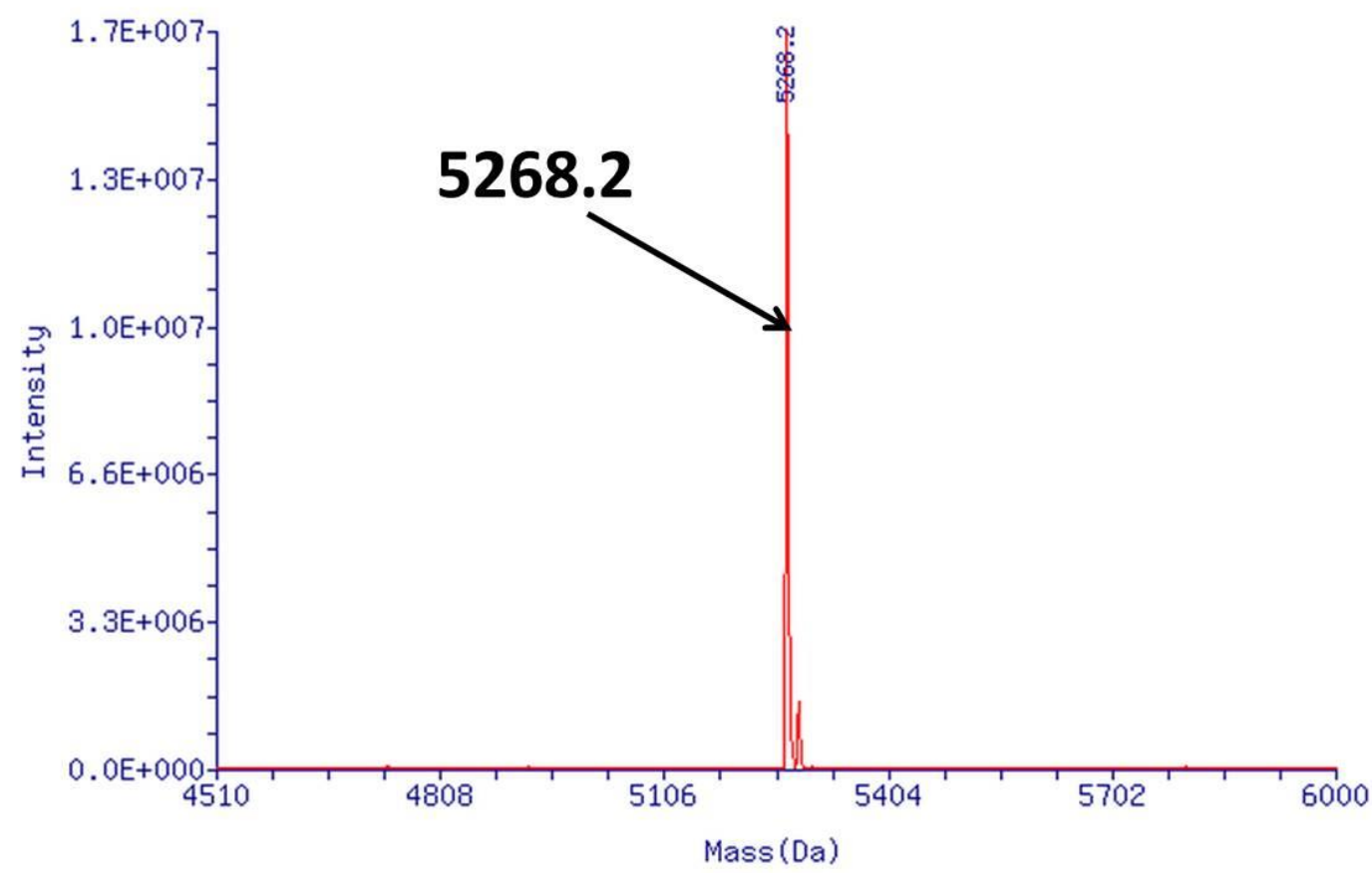

Figure S3. TOP shows the chemical structure of TPEPy-LDNA. Bottom provides the mass spectrum for TPEPy-LDNA, with the molecular weight 5268.2. 
2.4 Chemical structure and mass spectrum of FAM-LDNA and Cy3-MBDNA
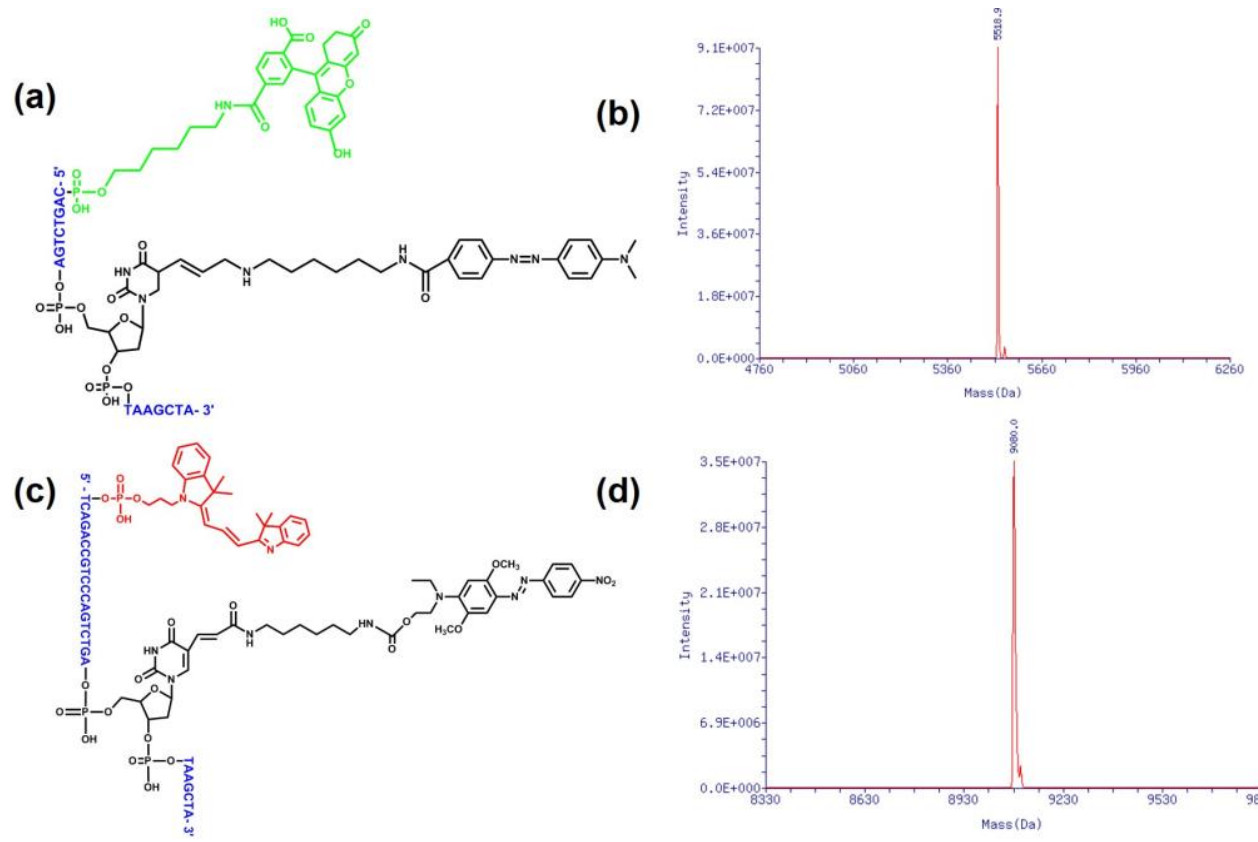

(d)

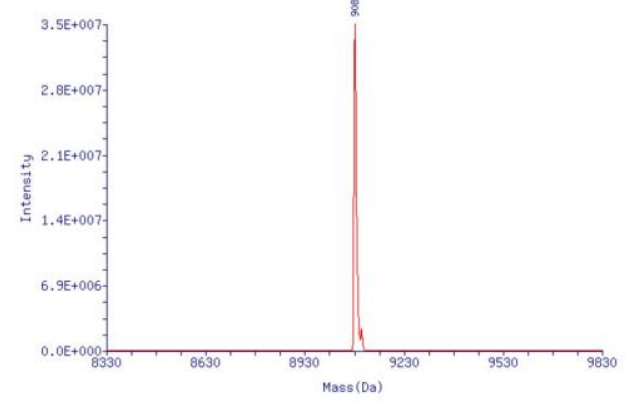

Figure S4. The chemical structure of FAM-LDNA (a) and Cy3-MBDNA (c). The mass spectrum for FAM-LDNA (b) and Cy3-MBDNA (d), with the molecular weight, 5518.9 and 9080.0 . 


\subsection{Light scattering measurement of liberated TPEPy from TPEPy-LDNA}
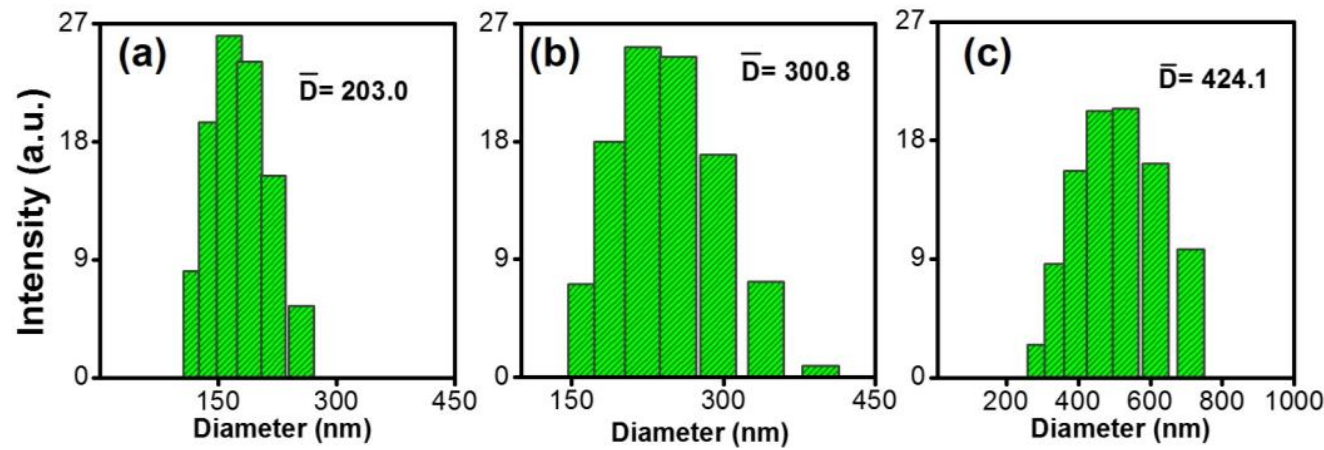

Figure S5. Dynamic light scattering measurements of the liberated TPEPy after inducing different concentrations (a) $10 \mathrm{pM}$, (b) $100 \mathrm{pM}$, and (c) $1 \mathrm{nM}$ miR-21 under the enzyme Exo III from TPEPy-LDNA $(10 \mu \mathrm{M})$. 


\subsection{The sequences of involved oligonucleotides}

Table S1. The sequences of involved oligonucleotides are as follows:

\begin{tabular}{|c|c|}
\hline Name & Sequence ( ${ }^{\prime}$ ' to $3^{\prime}$ ) \\
\hline miR-21 & UAG CUU AUC AGA CUG AUG UUG A \\
\hline R155 & UUA AUG CUA AUC GUG AUA GGG G \\
\hline R210 & CUG UGC GUG UGA CAG CGG CUG A \\
\hline R221 & A GCU ACA UUG UCU GCU GGG UUU C \\
\hline SM miR-21 & UAG CUU AUA AGA CUG AUG UUG A \\
\hline TM miR-21 & UAG CUU AUA ACC CUG AUG UUG A \\
\hline FAM-LDNA & (FAM) -CAG TCT GAT-(DABCYL) AAG CTA \\
\hline Cy3-MBDNA & (Cy3) - TCA GAC CGT CCC AGT CTG AT-(BHQ2) A AGC TA \\
\hline TPEPy-LDNA & TPEPy-CAG TCT GAT AAG CTA \\
\hline
\end{tabular}

The red shown in sequences reveal the mismatch part. 
2.7 Confocal images and three-dimensional map of MCF-7 cells stained with TPEPy-LDNA

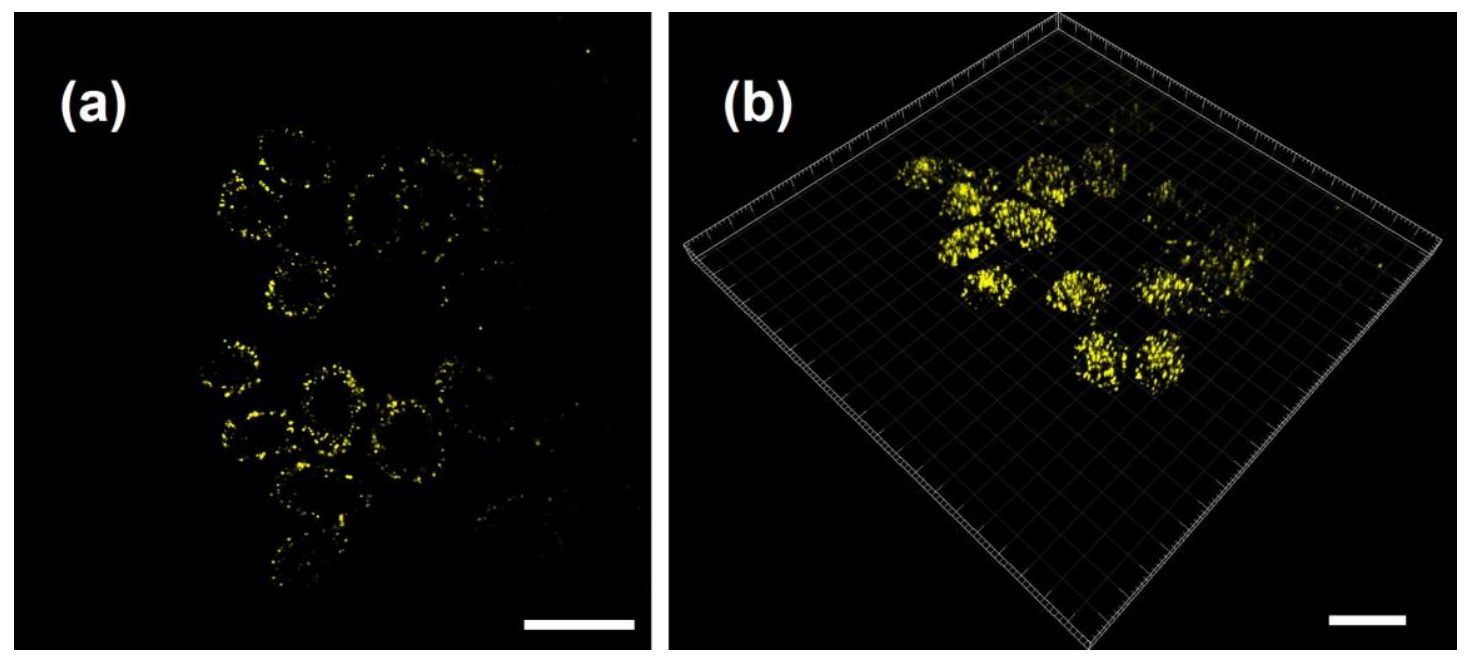

Figure S6. (a) Confocal microscopy images of MCF-7 cells stained with TPEPy-LDNA $(6.6 \mu \mathrm{M})$ for $60 \mathrm{~min}$. (b) Corresponding three-dimensional map of MCF-7 cells. $\lambda_{\text {ex }}=405 \mathrm{~nm}, \lambda_{\mathrm{em}}=560-620 \mathrm{~nm}$. Scale bar, $20 \mu \mathrm{m}$. 


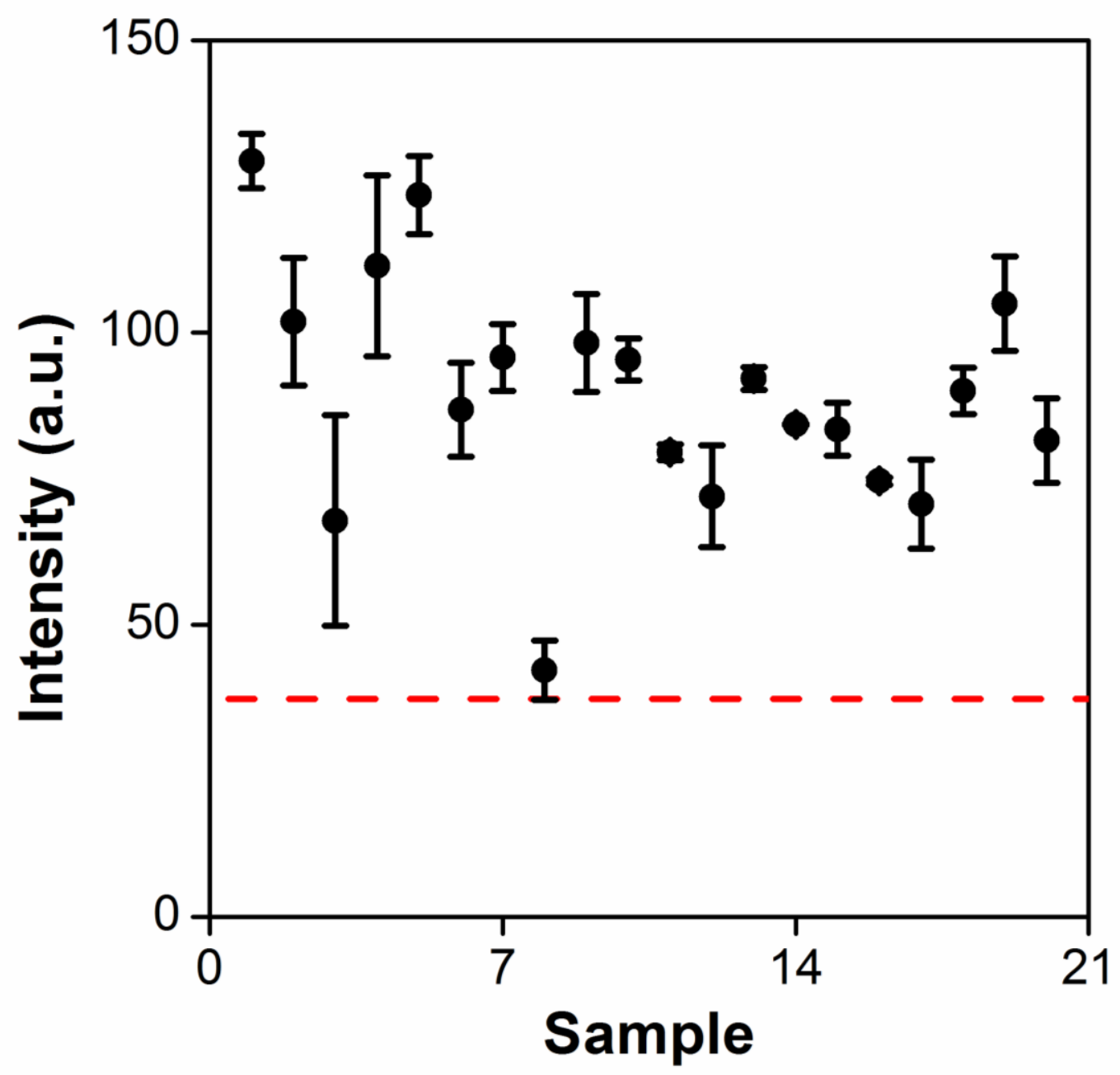

Figure S7. The detection of mir-21 in 20 clinical cancer patients' sample was performed by TPEPy-LDNA, which showed the good specificity of our assay. All of the data were higher than the threshold. The horizontal dashed line represents the threshold level according to the definition of $I_{0}+3 \sigma$ ( $I_{0}$ means average fluorescence intensity at $605 \mathrm{~nm}$ between 5 normal specimens, $\sigma$ means standard deviation of fluorescence intensity at $605 \mathrm{~nm}$ between 5 normal specimens.). 


\subsection{Corresponding disease information of the urine samples}

Table S2. Corresponding disease information of bladder cancer urine samples

\begin{tabular}{cccccccc}
\hline $\begin{array}{c}\text { Sample } \\
\text { No. }\end{array}$ & $\begin{array}{c}\text { Region } \\
\text { of } \\
\text { patient }\end{array}$ & $\begin{array}{c}\left(\mathbf{I / I} \mathbf{I}_{\mathbf{0}} \mathbf{- 1} \text { at }\right. \\
\mathbf{6 0 5} \mathbf{~ n m}\end{array}$ & Gender & Age & $\begin{array}{c}\text { Urine } \\
\text { apperance }\end{array}$ & Disease & Grade/Stage \\
\hline 1 & Jiangxi & 3.2937 & Male & 24 & Clear & Carcinoma & G1/NMIBC \\
2 & Jiangxi & 2.3810 & Female & 78 & Blood & Carcinoma & G2/MIBC \\
3 & Jiangxi & 1.2514 & Female & 56 & Clear & Carcinoma & G2/MIBC \\
4 & Jiangxi & 2.6975 & Male & 60 & Clear & Carcinoma & G3/MIBC \\
5 & Jiangxi & 3.1011 & Male & 51 & Blood & Carcinoma & G2/NMIBC \\
6 & Jiangxi & 1.8806 & Male & 54 & Blood & Carcinoma & G0/NMIBC \\
7 & Jiangxi & 2.1778 & Male & 77 & Clear & Carcinoma & G2/MIBC \\
8 & Jiangxi & 0.4021 & Female & 59 & Clear & Carcinoma & G1/NMIBC \\
9 & Jiangxi & 2.2602 & Male & 38 & Blood & Carcinoma & G1/NMIBC \\
10 & Jiangxi & 2.1660 & Female & 38 & Blood & Carcinoma & G1/NMIBC \\
11 & Jiangxi & 1.6389 & Female & 64 & Clear & Carcinoma & G0/NMIBC \\
12 & Jiangxi & 1.3886 & Female & 60 & Clear & Carcinoma & G3/MIBC \\
13 & Jiangxi & 2.0575 & Male & 44 & Blood & Carcinoma & G3/MIBC \\
14 & Jiangxi & 1.7973 & Male & 57 & Blood & Carcinoma & G2/NMIBC \\
15 & Jiangxi & 1.7696 & Male & 72 & Blood & Carcinoma & G3/MIBC \\
16 & Jiangxi & 1.4775 & Female & 41 & Clear & Carcinoma & G1/NMIBC \\
17 & Jiangxi & 1.3450 & Male & 43 & Clear & Carcinoma & G0/NMIBC \\
18 & Jiangxi & 1.9877 & Male & 35 & Clear & Carcinoma & G2/NMIBC \\
19 & Jiangxi & 2.4832 & Male & 53 & Blood & Carcinoma & G2/NMIBC \\
20 & Jiangxi & 1.7054 & Female & 68 & Blood & Carcinoma & G1/NMIBC \\
\hline
\end{tabular}

Table S3. Corresponding information of normal people

\begin{tabular}{cccccc}
\hline $\begin{array}{c}\text { Sample } \\
\text { No. }\end{array}$ & $\begin{array}{c}\text { Region of } \\
\text { normal } \\
\text { people }\end{array}$ & $\begin{array}{c}\boldsymbol{I} \text { at 605 } \\
\mathbf{n m}\end{array}$ & Gender & Age & $\begin{array}{c}\text { Urine } \\
\text { apperance }\end{array}$ \\
\hline 1 & Hubei & 27.1396 & Male & 26 & Clear \\
2 & Hubei & 30.9048 & Male & 28 & Clear \\
3 & Hubei & 27.5298 & Female & 23 & Clear \\
4 & Hubei & 31.8812 & Male & 21 & Clear \\
5 & Hubei & 33.1701 & Female & 30 & Clear \\
\hline
\end{tabular}



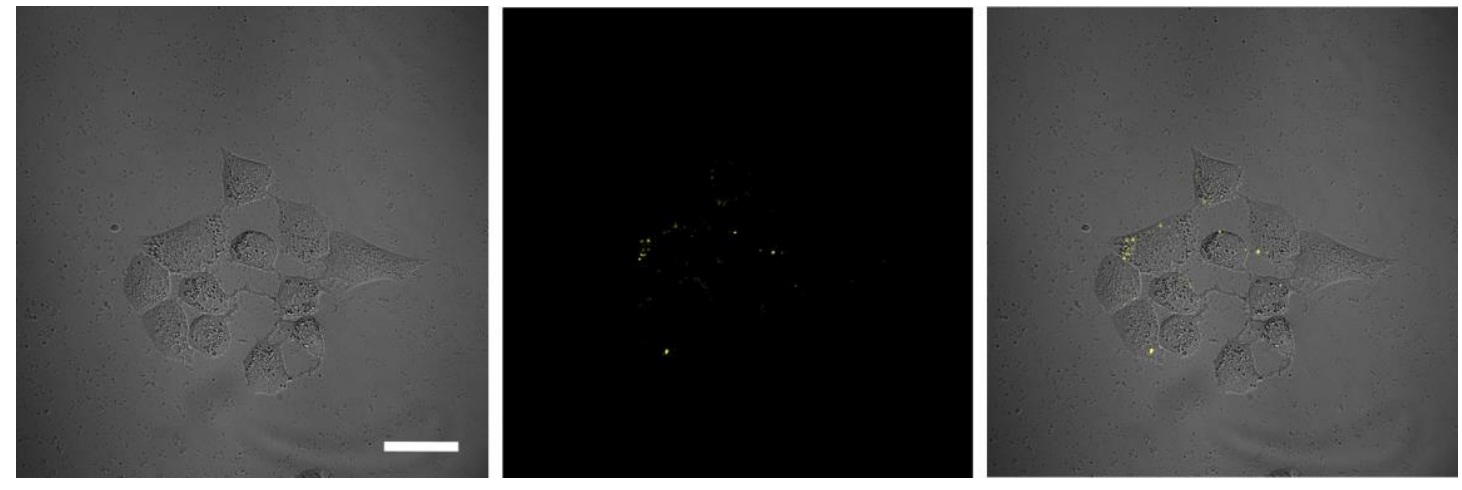

Figure S8. The negative control experiment, which shows very weak fluorescence intensity in the absence of Exo III, illustrates the enzyme is indispensable. The scale bar in the figure is $20 \mu \mathrm{m}$. 

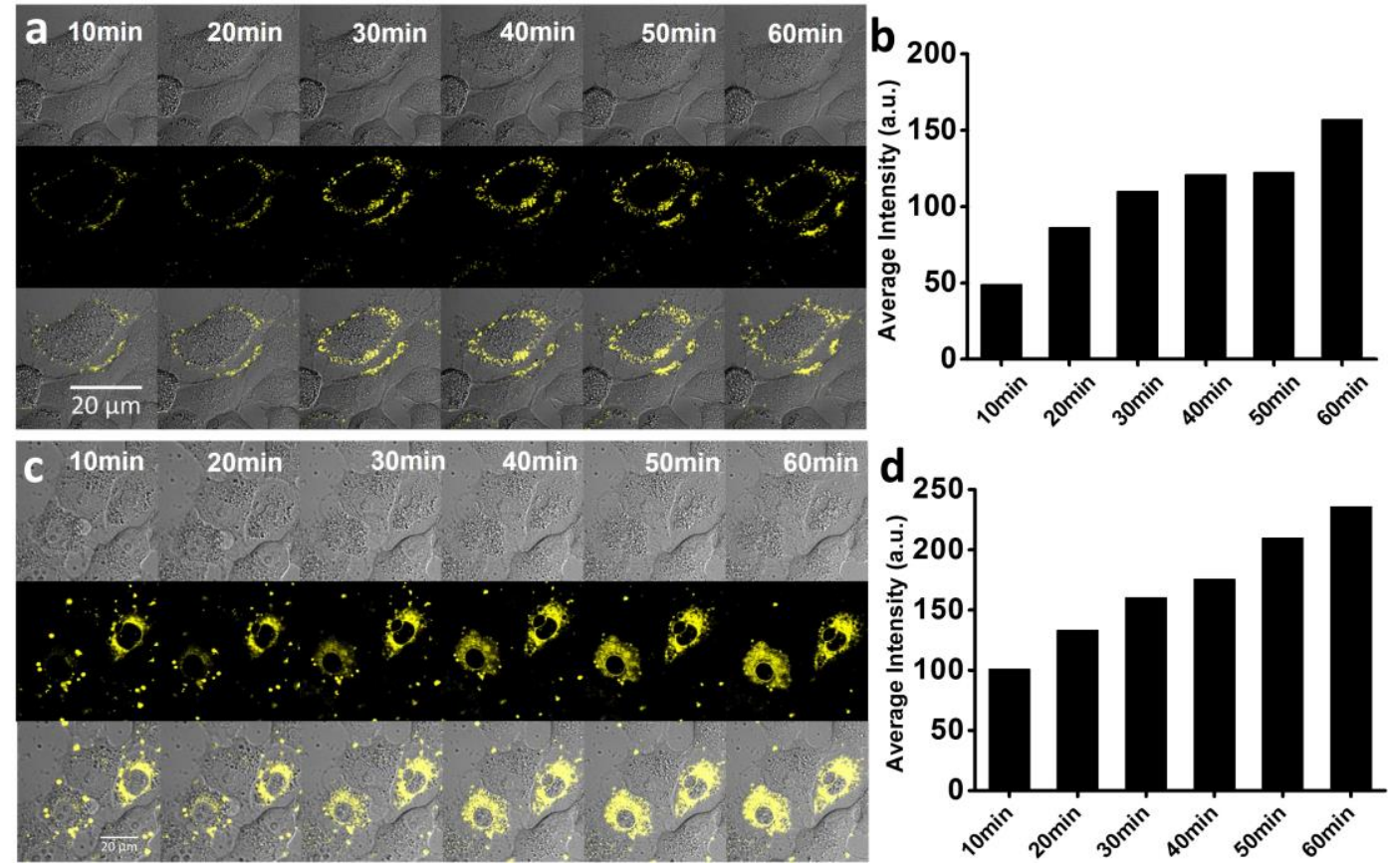

Figure S9. Time-dependent detection process of miR-21 in MCF-7 with TPEPy-LDNA $(6.6 \mu \mathrm{M})(\mathrm{a}, \mathrm{c})$. The signal $(\mathrm{b}, \mathrm{d})$ increased gradually within $60 \mathrm{~min}$ for TPEPy-LDNA in vivo. The scale bar in the figure is $20 \mu \mathrm{m}$. 

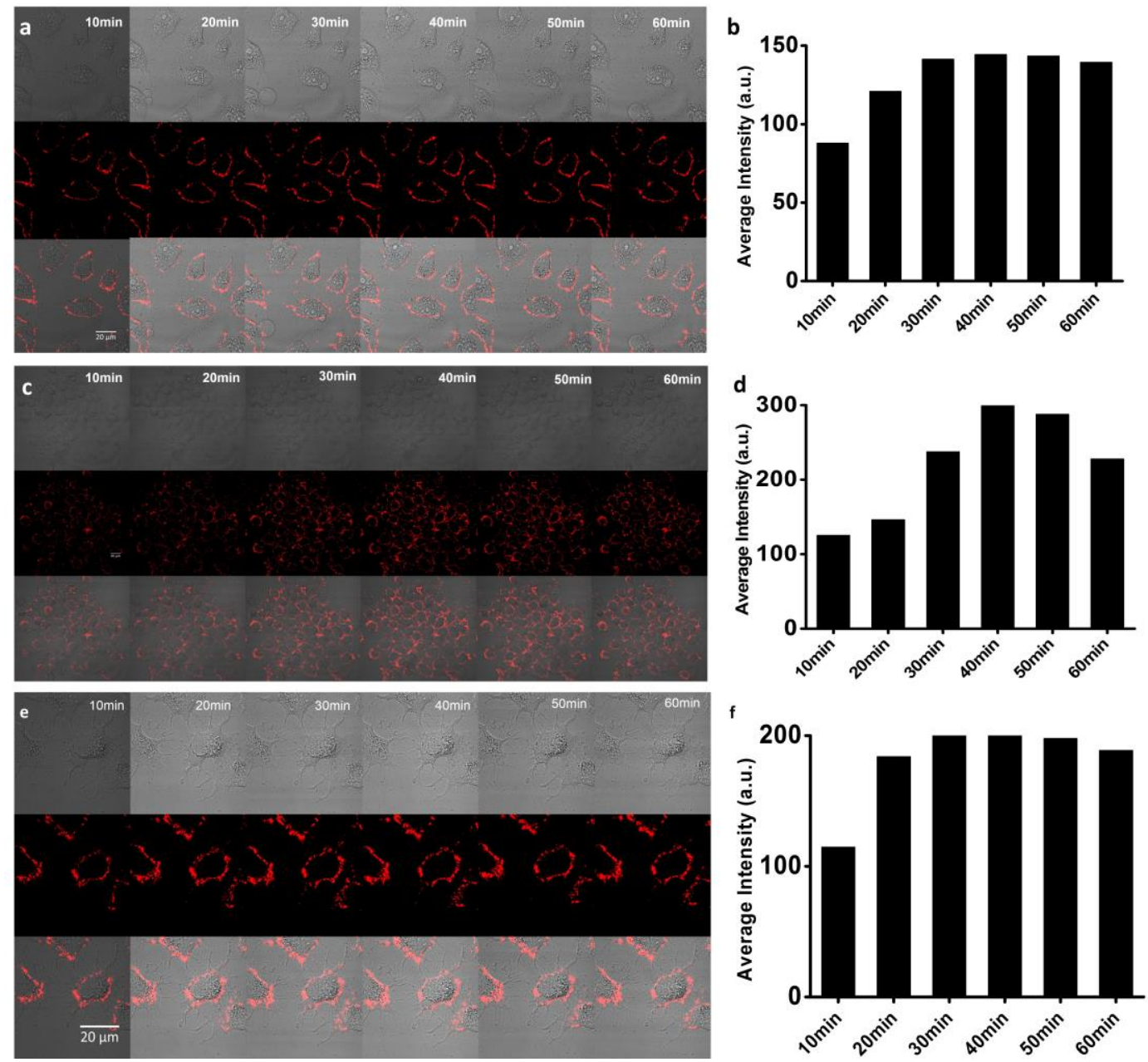

Figure S10 Time-dependent detection process of miR-21 in MCF-7 with Cy3-MBDNA (500 nM) (a, c, e), which showed totally different behaviors. The signal of Cy3-MBDNA (b, d, f) increased from $10 \mathrm{~min}$ to $50 \mathrm{~min}$, but began to fade at 60 min finally. The scale bar in the figure is $20 \mu \mathrm{m}$. 

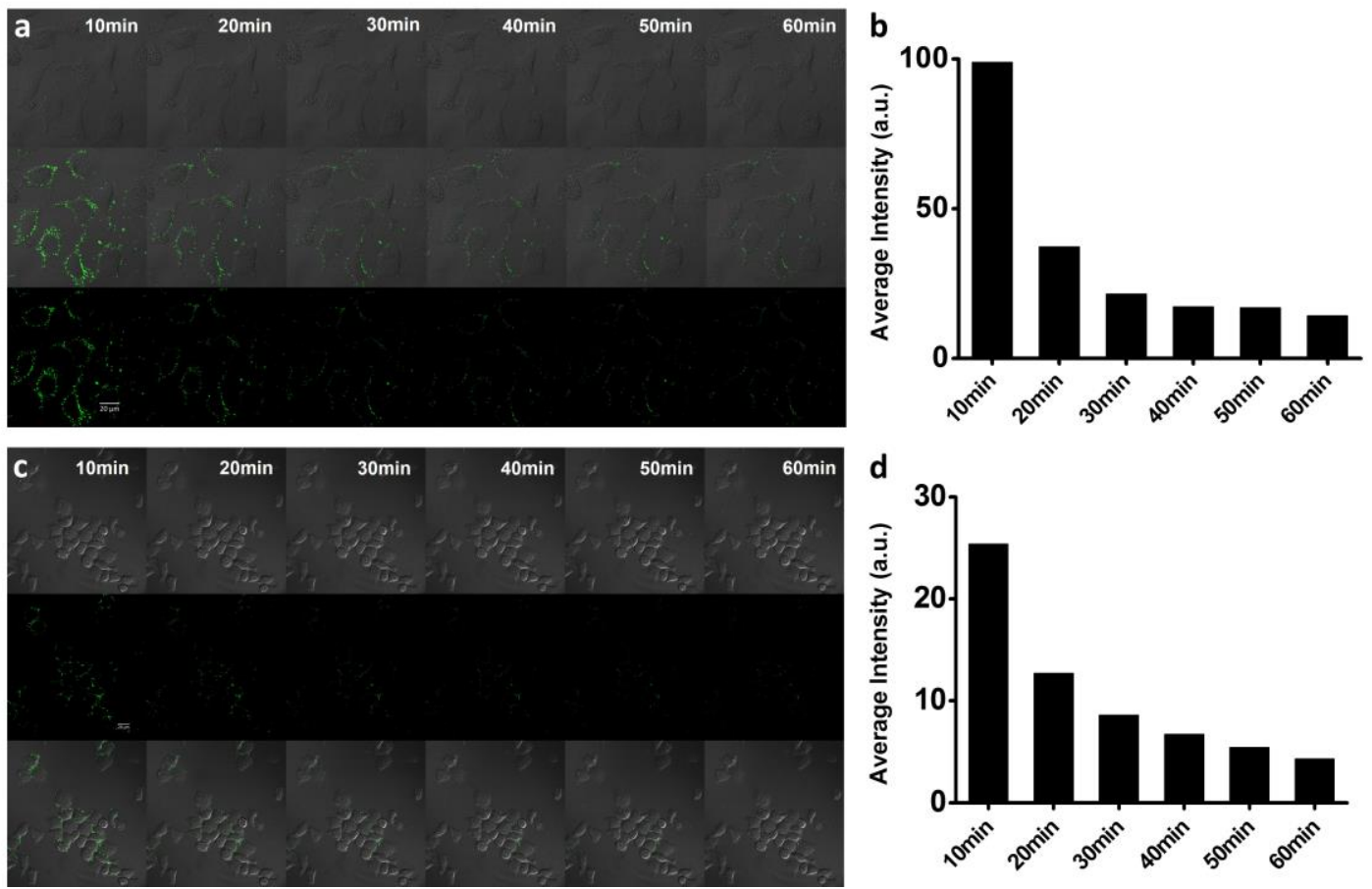

Figure S11 Time-dependent detection process of miR-21 in MCF-7 with FAM-LDNA $(500 \mathrm{nM})(\mathrm{a}, \mathrm{c})$, which showed totally different behaviors. The signal of FAM-LDNA (b, d) decreased from $10 \mathrm{~min}$ to $60 \mathrm{~min}$ in MCF-7. The scale bar in the figure is 20 $\mu \mathrm{m}$. 


\section{Reference}

(1) Shi, H.; Zhao, N.; Ding, D.; Liang, J.; Tang, B. Z.; Liu, B. Fluorescent Light-up Probe with Aggregation-Induced Emission Characteristics for in Vivo Imaging of Cell Apoptosis, Org. Biomol. Chem. 2013, 11, 7289-7296.

(2) Min, X.; Zhuang, Y.; Zhang, Z.; Jia, Y.; Hakeem, A.; Zheng, F.; Cheng, Y.; Tang, B. Z.; Lou, X.; Xia F, Lab in a Tube: Sensitive Detection of MicroRNAs in Urine Samples from Bladder Cancer Patients Using a Single-Label DNA Probe with AIEgens ACS Appl. Mater. Interfaces 2015, 7, 16813-16818.

(3) Zhang, S.; Metelev, V.; Tabatadze, D.; Zamecnik, P. C.; Bogdanov, A. Fluorescence Resonance Energy Transfer in Near-Infrared Fluorescent Oligonucleotide Probes for Detecting Protein-DNA Interactions. Proc. Natl. Acad. Sci. U.S.A. 2008, 105, 4156-4161.

(4) Chen, Z.; Ji, M.; Hou, P.; Lu, Z. Exo-Dye-based Assay for Rapid, Inexpensive, and Sensitive Detection of DNA-binding Proteins. Biochem. Biophys. Res. Commun. 2006, 345, 1254-1263. 